International Journal of Advanced Trends in Computer Science and Engineering

Available Online at http://www.warse.org/IJATCSE/static/pdf/file/ijatcse2991.42020.pdf

https://doi.org/10.30534/ijatcse/2020/2991.42020

\title{
Variations of ionospheric F2 layer critical frequency in Malaysia during different solar phases
}

\author{
Noreen Nabilla Risal ${ }^{1}$, Mariyam Jamilah Homam ${ }^{2 *}$, Rohaida Mat Akir $^{3}$ \\ ${ }^{1,2,3}$ Wireless and Radio Science Centre, Faculty of Electrical and Electronic Engineering, \\ Universiti Tun Hussein Onn Malaysia, Malaysia \\ 2 mariyam@uthm.edu.my
}

\begin{abstract}
This study investigated the variations in ionospheric F2 layer critical frequency (foF2) and sunspot number during different solar epochs (2008 and 2011). The foF2 data were obtained from an ionosonde installed at the Wireless and Radio Science Centre, Universiti Tun Hussein Onn Malaysia, Johor $\left(1.86^{\circ} \mathrm{N}, 103.80^{\circ} \mathrm{E}\right)$. The dependence of foF2 during low and medium solar activities was analysed. The highest and lowest foF2 were $6.55 \mathrm{MHz}$ at 1200 universal time (UT) (2000 local time (LT)) and $2.34 \mathrm{MHz}$ at $2100 \mathrm{UT}(0500 \mathrm{LT})$, respectively, from the observations during hourly diurnal variations of foF2 in 2008. The highest and lowest foF2 were $7.59 \mathrm{MHz}$ at $1000 \mathrm{UT}(1800 \mathrm{LT})$ and $3.24 \mathrm{MHz}$ at $0600 \mathrm{UT}$ (1400 LT), respectively, in 2011. The highest and lowest foF2 were 5.65 and $3.07 \mathrm{MHz}$, respectively, during monthly variations of foF2 in 2008, whereas the highest and lowest foF2 were 6.26 and $6.06 \mathrm{MHz}$, respectively, in 2011. The trends in 2008 and 2011 show that foF2 slowly increases in the morning and reaches its peak at noon. foF 2 reaches its lowest frequency from dusk to midnight with the decrease in solar activity during night time.
\end{abstract}

Key words: Critical frequency, F2 layer, Ionosphere, Solar activity, Sunspot number

\section{INTRODUCTION}

The ionosphere is the ionised part of the Earth's upper atmosphere that has practical importance because it can affect radio propagation to distant places on the Earth. However, it is highly variable because of the influence of solar, geomagnetic, and other factors. Solar activity indicators, such as sunspot numbers (SSNs), are used as indirect indicators of ultraviolet (UV) solar and X-ray emissions that are mainly responsible for causing ionisation in the Earth's upper atmosphere.

The F2 layer critical frequency, foF2, is an important ionospheric parameter influenced by local time, geographical latitude, solar and magnetic activities, atmospheric background wind and other factors [1]. Several studies have been conducted to observe the changes in critical frequency and related them to other parameters, including solar activities [2-7]. The SSN appears on the sun's surface with an average

cycle of approximately 11 years. An 11-year solar cycle (SC) is an influential change in solar activity that has a significant impact on the equatorial ionosphere [7]. The relationship between foF 2 and some solar activity indices has been studied at the span of 11-year SC (1985 to 1995) with maximum activity observed at the middle of the cycle [6].

Solar radio flux at 10.7 (F10.7) and SSN are common solar activity indices used by many researchers in [2-7]. This study only focuses on the SSN. The relationship between foF2 and SSN is approximately linear [8]. The variability of foF2 and its relationship to SSN during low (2008) and medium solar activities (2011) were presented in this study. The diurnal and monthly variations of foF 2 were investigated.

\section{RELATIONSHIP OF CRITICAL FREQUENCY AND SSN}

Numerous studies have been conducted to examine the relationship between foF 2 and solar activity indices, such as F10.7 and SSN. Most studies developed neural network model to evaluate the relationship between solar activity and foF2. Neural network is one of the Artificial Intelligence (AI) areas. AI refers to the wide computer technology that enables human needs to be fulfilled including human intelligence, decision-making analytics, pattern recognition, visual \& speech recognition, and language translation using a subclass algorithm within the artificial intelligence framework [9]. Neural network is inspired by the simplified human brain mechanism that has been commonly used to forecast [10][11]. However, our topic is focusing on predicting foF2. The variability of foF2 in equatorial regions under SC 20 (1976 to 1986) and SC 21 (1986 to 1996) during minima and maxima phases were investigated in [2]. The researchers reported the foF2 variabilities in two equatorial regions, namely, Ouagadougou $\left(12.40^{\circ} \mathrm{N}, 358.5^{\circ} \mathrm{E}\right)$ and Manila $\left(14.60^{\circ} \mathrm{N}\right.$, $120.98^{\circ} \mathrm{E}$ ). 
The researchers in [2] highlighted the variability of foF2 across different seasons, days, times, solar events and latitude for better analysis. Their results showed significant similarities between foF2 seasonal variations at time intervals from 1976 to 1996 . The foF2 in the Manila station at daytime is higher than that in the Ouagadougou station because the Manila station is closer to the equatorial ionisation crest region. They concluded that the magnitude of foF 2 increases during the period of high solar activity, whereas it decreases during the period of low solar activity.

The conclusion of this study is supported by the findings in [3] that investigated the variability of foF2 in West Africa using ionosonde stations in Ouagadougou and Dakar $\left(14.71^{\circ} \mathrm{N}\right.$, $\left.342.53^{\circ} \mathrm{E}\right)$ from 1979 to 1986 during low, moderate and high solar activities. The observed results of foF 2 were compared under diurnal and seasonal variations. foF 2 increases with the increase in solar activity on the basis of foF 2 trend.

The researchers in [4] agree with the findings of [3]. In [4] the variations of foF2 were investigated and compared diurnally and seasonally. They used ionosonde data from Parit Raja, Malaysia station $\left(1.86^{\circ} \mathrm{N}, 103.80^{\circ} \mathrm{E}\right)$, which is close to the geomagnetic equator with a geographic latitude of $5^{\circ} \mathrm{N}$. The dependence of critical frequency from 2005, 2006 and 2007 under the solar minimum period of $23 \mathrm{SC}$ was analysed. The highest foF2 was observed in 2005 because of the high number of SNNs. The highest annual averages of SSNs are 29.8, 15.2 and 7.5 in 2005, 2006 and 2007, respectively.

The researchers in [5] and [6] studied the monthly median values of foF2 in Okinawa $\left(26.30^{\circ} \mathrm{N}, 127.80^{\circ} \mathrm{E}\right)$. In [5], the monthly median foF2 variations for 12 months from 2003 to 2010 starting from moderate to low SSN were evaluated. The value of foF2 increases in years with moderate SSN starting from sunrise to midnight on the basis of the observations on the monthly median value of foF2 in [5]. In [6], the relationship between foF 2 and solar activity indices of solar activity at three low-latitude ionosphere stations during SC 22 (1985 to 1996) was explored, and the trend of foF2 follows a similar trend as SSN, indicating that the higher the SSN is, the higher the value of foF 2 will be.

A strong dependence was observed between the solar activity of foF2 during SC 22 in a comparative study between foF2 and SSN [7]. The data from Ouagadougou station were used, similar to [2] and [3]. In [7], the solar activity dependence of foF2 is strong in years with moderate solar activity, whereas it decreases in the SC within years with high and low solar activities.

\section{METHODOLOGY}

In the present study, the foF 2 data were obtained from an ionosonde installed at the Wireless and Radio Science Centre,
Universiti Tun Hussein Onn, Johor. The ionosonde has been in operation since 2004. The recoded SSN data were obtained from the monthly SSNs of the Bureau of Meteorology, Australian Government, National Geophysical Data Centre of the US National Oceanic \& Atmospheric Administration and SSNs in Solar Influences Data Analysis Centre, as illustrated in Figure 1.

A peak in the SSN is called the solar maxima where the sunspot count is high, whereas a peak in the SNN with few sunspots is called the solar minima. The monthly SSNs from March to December in 2008 and 2011 are listed in Table 1, where the average of SSN in 2008 is 3.82, which is lower than that in 2011, which is 78.72. Year 2008 is the period of low solar activity, whereas 2011 is the period of medium solar activity.

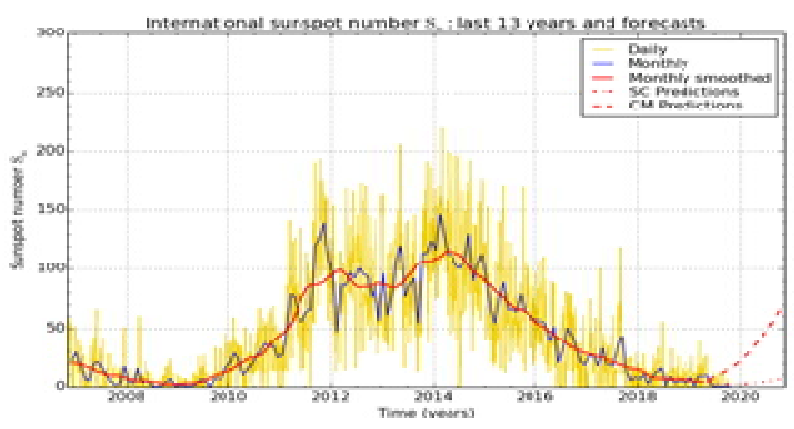

Figure 1: SSNs from 2007 to 2020 [12]

Table 1: Monthly SSNs for 2008 and 2011 [12]

\begin{tabular}{|c|c|c|}
\hline Month & $\mathbf{2 0 0 8}$ & $\mathbf{2 0 1 1}$ \\
\hline March & 5.1 & 53.8 \\
\hline April & 5.1 & 61.1 \\
\hline May & 5.3 & 69.3 \\
\hline June & 4.8 & 77.2 \\
\hline July & 4.0 & 83.6 \\
\hline August & 3.8 & 86.3 \\
\hline September & 3.2 & 86.6 \\
\hline October & 2.4 & 87.4 \\
\hline November & 2.3 & 89.4 \\
\hline December & 2.2 & 92.5 \\
\hline
\end{tabular}

foF 2 was observed for a period of 10 months during 2008 and 2011. The diurnal result is based on 24-hour observations from March to December in these years. Statistical analysis using the average value from observations showed the trend of foF2 during the observed periods and cycles and the behavior of foF 2 in response to different solar phases. The monthly variations in these periods were discussed. The observations of foF2 dependency to SSN and its relation to foF2 in 2011 may be limited because the foF 2 data in 2008 are limited. 
Noreen Nabilla Risal et al., International Journal of Advanced Trends in Computer Science and Engineering, 9(1.4), 2020, 198 - 202

\section{RESULT AND DISCUSSION}

\subsection{Diurnal variations of critical frequency during} different solar phases

The comparison of critical frequencies, foF 2 from March to December in 2008 and 2011 (0000 to 2300 UT) is shown in Figure 2. The hourly diurnal variation of foF 2 for each month
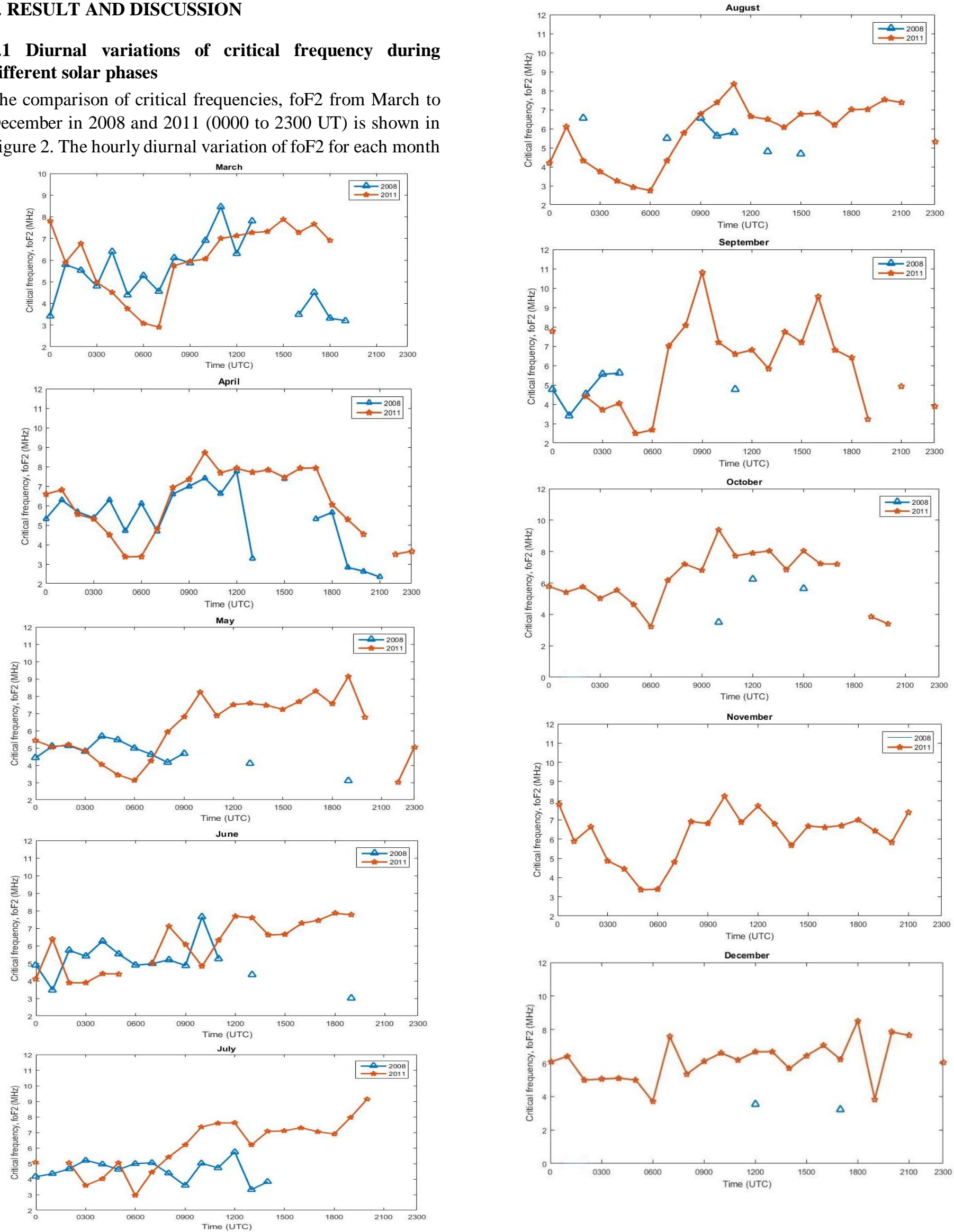

Figure 2: Hourly diurnal variations of critical frequency for each month from March to December in 2008 and 2011 
from March to December in Figure 2 is similar with the trend of hourly diurnal foF2 in 2008 and 2011, as shown in Figure 3. The local time (LT) is $8 \mathrm{~h}$ ahead from UT. As shown in Figure 3, the highest and lowest foF2 are $8.47 \mathrm{MHz}$ at 1100 UT (1900 LT) and $3.03 \mathrm{MHz}$ at $1900 \mathrm{UT}$ (0300 LT), respectively, in March 2008, whereas the highest and lowest foF2 are $10.80 \mathrm{MHz}$ at $0900 \mathrm{UT}(1700 \mathrm{LT})$ and $2.49 \mathrm{MHz}$ at 0500 UT (1300 LT), respectively, in September 2011.

As shown in Figure 2, the trend of foF2 slowly increases in the early morning, which is $7.81 \mathrm{MHz}$ at $0000 \mathrm{UT}$ (0800 LT) during March 2011 and reaches its highest frequency from noon to dusk within 1200 to 2100 UT (2000 to 0500 LT). Compared with the result of [4], the foF 2 trend in the present study slowly increases in the early morning, which is 6.75 MHz from 0000 UT to 0100 UT (0800 to 0900 LT), reaches its maximum from noon to late evening, and decreases at night. This result is supported by the findings in [5], which stated that the value of foF2 increases early in the morning at 1000 UT (0600 LT) or from sunrise to midnight.

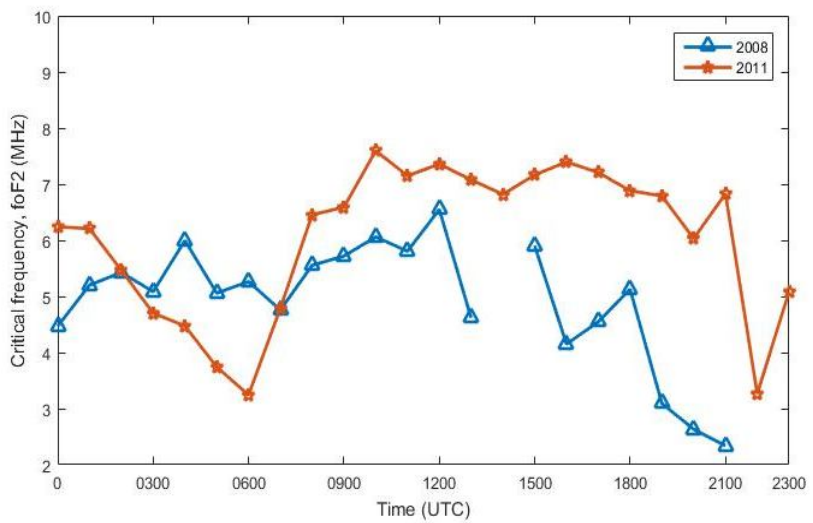

Figure 3: Hourly diurnal variation of foF 2 in 2008 and 2011

Solar activities increase during daytime, thereby increasing the ionised layer in the ionosphere and enabling high frequencies to propagate over long distances [13]. The foF2 reaches its low values from $4.53 \mathrm{MHz}$ to $3.51 \mathrm{MHz}$ at 2000 to 2300 UT (0400 to 0700 LT) at night because of low solar activity. This trend corresponds with the result of [4], where foF2 decreases from 6.8 MHz to 4.2 MHz from 1630 to 2330 UT (0030 to 0730 LT).

The trend of foF2 variations in 2008 and 2011 is approximately similar, as shown in Figure 3. foF2 slowly increases early in the morning, reaches its peak from noon to dusk, and slowly decreases to low frequency at night. The highest critical frequencies (foF2) are $6.55 \mathrm{MHz}$ at $1200 \mathrm{UT}$ (2000 LT) and $7.59 \mathrm{MHz}$ at $1000 \mathrm{UT}$ (1800 LT), whereas the lowest foF2 are $2.34 \mathrm{MHz}$ at $0900 \mathrm{UT}$ (1700 LT) and 3.24 MHz at 0600 UT (1400 LT) in 2008 and 2011, respectively.

\subsection{Monthly variations of critical frequency during different solar phases}

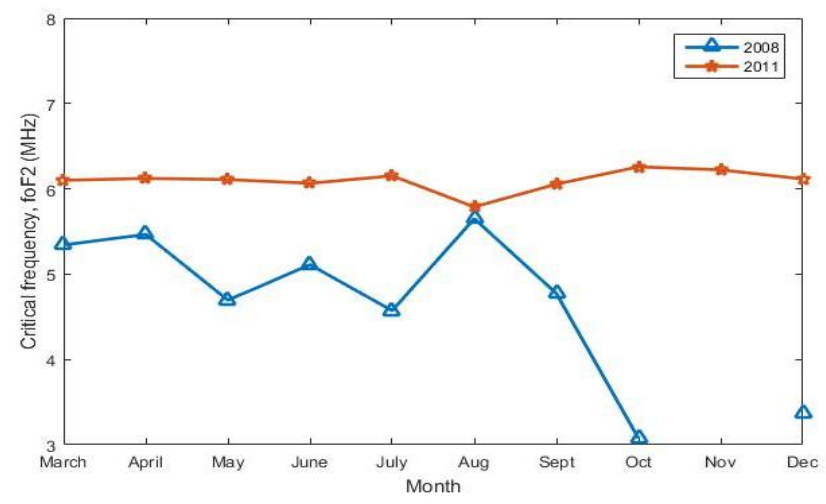

Figure 4: Monthly variation of foF2 in 2008 and 2011

As shown in Figure 4, the foF2 during March equinox is 6.10 $\mathrm{MHz}$ in the year with medium solar activity (2011). Then, the foF2 slightly increases and decreases until May before reaching its lowest at around the June solstice. The analysis of [7] showed that the foF 2 in the June solstice reaches it minimum value because of the maximum sun-Earth distance (short daytime solar radiation influx) in the Northern Hemisphere during this season rather than the trend in solar activity. Therefore, foF2 slightly increased in July and decreased to its minimum during the September equinox.

As shown in Figure 4, the critical frequency reaches 5.34 MHz in March with low solar activity (2008). Then, the foF2 decreases to $4.57 \mathrm{MHz}$ in July and becomes slightly unstable between May and June. Compared with the results of [7], the current study shows the same pattern for foF2, where it increases in the early March equinox and slowly decreases from the maximum until the end of the June solstice. foF2 increases to $5.65 \mathrm{MHz}$ before slowly decreasing in December. In [7], foF 2 increases from the end of the June solstice and decreases through the December solstice. The monthly variations of foF2 and SSN in 2008 and 2011 are shown in Figure 5.

\section{CONCLUSION}

This study concluded that SSN has a large influence on the variation of foF 2 in different solar phases. The highest and lowest frequencies of foF 2 under hourly diurnal variation are 6.55 and $2.34 \mathrm{MHz}$ and 7.59 and $3.24 \mathrm{MHz}$ during low (2008) and medium solar activities (2011), respectively. The highest and lowest frequencies of foF 2 under monthly variations are 5.65 and $3.07 \mathrm{MHz}$ and 6.26 and $6.06 \mathrm{MHz}$, respectively, during low (2008) and medium solar activities (2011). These findings show that the foF 2 of the ionosphere is higher during medium solar activity compared with low solar activity. 

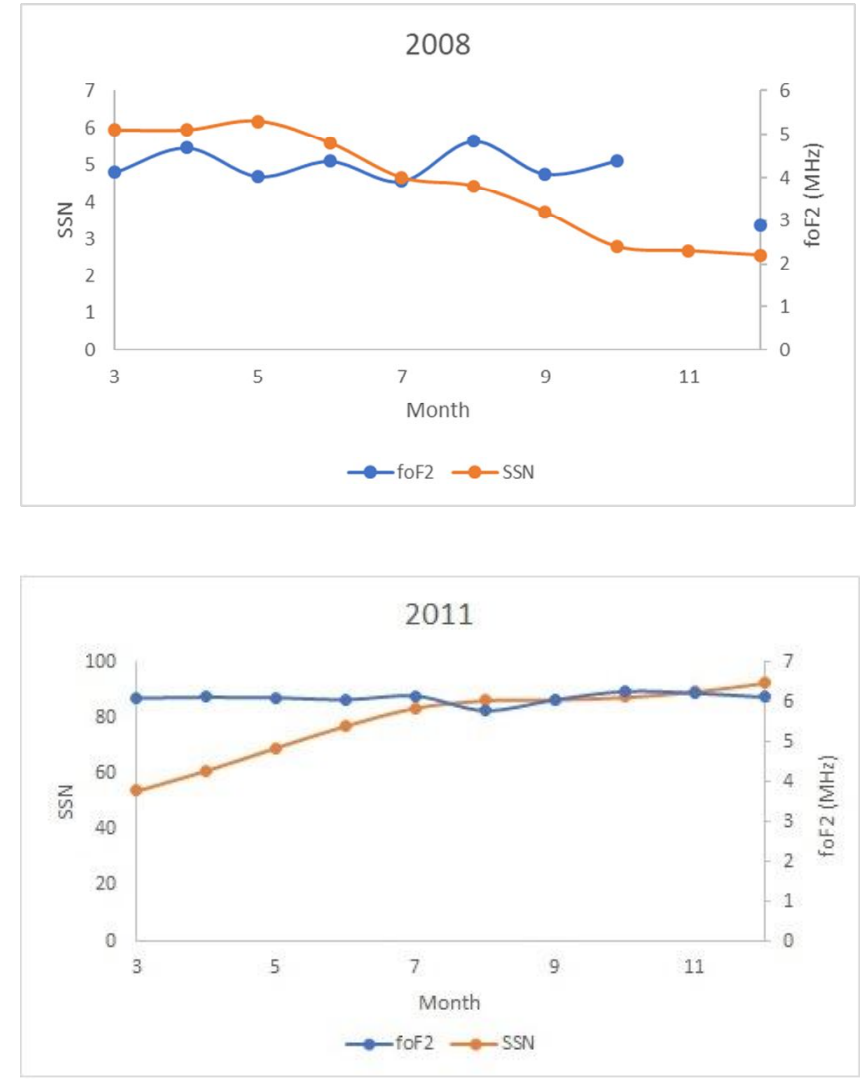

Figure 5: Monthly variations of foF2 and SSN in 2008 and 2011

\section{ACKNOWLEDGEMENT}

The authors would like to thank the Ministry of Education Malaysia for supporting this research under Fundamental Research Grant Scheme Vot No. FRGS/1/2017/STG09/UTHM/02/1 and partially sponsored by Universiti Tun Hussein Onn Malaysia. The authors also appreciate the World Data Centre (WDC) and the WDC-SILSO for providing the sunspot data.

\section{REFERENCES}

[1] C. Chen, S. Sun and B. Panpan, "Forecasting the ionospheric foF2 in Chinese region by neural network technique," Proc. 9th Int. Symp. Antenna, Propag. EM Theory, Guangzhou., vol. 2, pp. 399-402, 2010.

[2] M. Kaboré, J. L. Zerbo, C. Zoundi and F. Ouattara, "Variability of the critical frequency foF2 for equatorial regions during solar cycle's minima and maxima at Ouagadougou and Manila stations," Int. J. Geosci., vol. 10, pp. 833-844, 2019.

[3] O.R. Salami and E.F. Nymphas, "Variability of the critical frequency of the F2 layer, foF2 in West Africa using ionosonde stations at Ouagadougou and Dakar," Res. J. Appl. Sci., vol. 7, no. 9-12, pp. 474-480, 2012.

[4] S. Abdullah and A.F.M. Zain, "Diurnal and seasonal variation of critical frequency in Malaysia from 2005 to 2007," Applied Mech. Mater., vol. 225, pp. 448-452, 2012.
[5] N.M.R. Al-Ubaidi, H.H.A. Al-Dahlaki and Z. Fadel, "Studying the variation of ionospheric parameters with earth geographic latitudes and sunspot number," Int. J. Sci. Nat., vol. 9, no. 1, pp. 52-59, 2018.

[6] S.A. Jilani, K. Khursheed and K. Mukhtar, "Comparative study of F2 Layer critical frequency: Solar Cycle 22," A Phys. Comput. Sci., vol. 55, no. 1, pp. 35-46, 2018.

[7] S.O. Ikubanni, B.O. Adebesin, S.J. Adebiyi and J.O. Adeniyi, "Relationship between F2 layer critical frequency and solar activity indices during different solar epochs," Indian J. Radio Sp. Phys., vol. 42, pp. 73-81, 2013.

[8] J.M. Roy, "Correlation between noon foF2 and sunspot number," IETE J. Res., vol. 2, no. 1, pp. 2-5, 1955. https://doi.org/10.1080/03772063.1955.11485936

[9] N. Nawaz, "Artificial intelligence interchange human intervention in the recruitment process in Indian software industry," Int. J. Adv. Trends Comput. Sci. Eng., vol. 8, no. 4, pp. 1433-1442, 2019.

[10] Nancy, B. Kumar, S. Waraich, and N. Kaur, "Hybridization of GA and Backpropagation for load balancing in grid system," Int. J. Adv. Trends Comput. Sci. Eng., vol. 2, no. 3, pp. 36-38, 2013.

[11] M.K. Singla, J. Gupta, P. Nijhawan, and A.S. Oberoi, "Electrical load forecasting using machine learning," Int. J. Adv. Trends Comput. Sci. Eng., vol. 8, no. 3, pp. 615-619, 2019. https://doi.org/10.30534/ijatcse/2019/45832019

[12] SILSO, World Data Center - Sunspot Number and Long-term Solar Observations, Royal Observatory of Belgium, on-line Sunspot Number catalogue: http://sidc.be/silso/dayssnplot.

[13] R.A. Malik, M. Abdullah, S. Abdullah and M.J. Homam, "The influence of sunspot number on high frequency radio propagation," 2014 IEEE Asia-Pacific Conf. Appl. Electromagn., pp. 107-110, 2015.

[14] R. Lukianova and K. Mursula, "Changed relation between sunspot numbers, solar UV / EUV radiation and TSI during the declining phase of solar cycle 23," $J$. Atmos. Solar-Terrestrial Phys., vol. 73, no. 2-3, pp. 235-240, 2011.

[15] R. Chattopadhyay, "Covariation of critical frequency of F2-layer and relative sunspot number," Bull. Astr. Soc. India, vol. 28, pp. 657-663, 2000.

[16] Y. Erdal, A. Kilcik, A.G. Elias, D. Burcin, O. Atila, V.B. Yurchyshyn and J.P. Rozelot, "Critical frequencies of the ionospheric F1 and F2 layers during the last four solar cycles: sunspot group type dependencies '," $J$. Atmos. Solar-Terrestrial Phys., vol. 171, no. November, pp. 157-163, 2018. https://doi.org/10.1016/j.jastp.2017.11.018 